EPJ Web of Conferences 53, 04001 (2013)

DOI: $10.1051 /$ epjconf/20135304001

(C) Owned by the authors, published by EDP Sciences, 2013

\title{
HiRes and TA spectrum measurements
}

\author{
Douglas R. Bergman ${ }^{a}$ for the HiRes and Telescope Array collaborations ${ }^{b}$ \\ Dept. of Physics and Astronomy, University of Utah, Salt Lake City, UT 84112, USA
}

\begin{abstract}
The High Resolution Fly's Eye and Telescope Array experiment are separate experiments, although there is significant overlap in both equipment and personnel. Despite using different measurement techniques the two experiments provide nearly identical measurements of the UHECR cosmic ray spectrum, with agreement in overall flux level and the positions of the spectral features of the Ankle and the GZK Cutoff. This similarity arises despite using entirely different detector techniques. What unites the measurements from the two experiments is the experimental methodology of a thoroughgoing use of Monte Carlo detector simulations which are required to produce distributions of observables which are the same as those in the actual data. The detector simulation is then used to estimate exposures and acceptances which are not directly measurable. The procedure of verification through data/MC comparisons removes many of the systematic errors associate with model assumptions inherent in the MC simulation.
\end{abstract}

\section{HIRES MONOCULAR MEASUREMENTS}

The High Resolution Fly's Eye Experiment (HiRes) operated from 1997-2006 on Dugway Proving Grounds in Utah. It measured extensive air showers (EASs) from ultra-high energy cosmic rays (UHECRs) using the fluorescence method. It has been described in greater detail previously [1, 2]. The experiment consisted of two sites, HiRes-I and HiRes-II, separated by $12.6 \mathrm{~km}$. The separation was chosen to provide a large aperture for viewing EASs in stereo. In this presentation, however, only the monocular results from each site acting separately will be discussed. The HiRes Collaboration included scientists from Columbia University, Los Alamos National Laboratory, the University of Montana, the University of New Mexico, Rutgers University, the University of Tokyo and the University of Utah.

Each of the two HiRes sites consisted of fluorescence telescopes. The telescopes used a 5.2- $\mathrm{m}^{2}$ primary mirror to collect fluorescence light which was focused onto a $16 \times 16$ array of photomultiplier tubes (PMTs) in the focal plane. Each PMT views 1 square degree of the sky giving the telescope an instrumented field-of-view of $14^{\circ} \times 16^{\circ}$. These telescopes were arranged in rings covering nearly the full $360^{\circ}$ in azimuth at each site. At HiRes-I there was one ring of telescopes covering from $3^{\circ}-17^{\circ}$ in elevation, while at HiRes-II there were two rings covering $3^{\circ}-31^{\circ}$.

The analysis of the data begins with a pattern matching stage which looks for nearly coincident signals in a set of contiguous PMTs within each telescope and in neighboring telescopes. The PMT signals should for a line in both space (angle on the sky) and in time (successive signals separated by fractions of a microsecond from one tube to the next). The spatial correlation of the tube pointing directions for cosmic ray events is used to define the Shower-Detector Plane (SDP) containing the line of the shower core and the point of the detector. For observations in stereo mode, the intersection of the two SDPs, one from each site, completely defines the shower geometry. For observations in monocular mode, the relative timing of the PMT signals must be used. Assuming the the shower front moves at the

\footnotetext{
ae-mail: bergman@physics .utah.edu

${ }^{b}$ For the full authorlist see Appendix "Collaborations" in this volume.
}

This is an Open Access article distributed under the terms of the Creative Commons Attribution License 2.0, which permits unrestricted use, distribution, and reproduction in any medium, provided the original work is properly cited. 

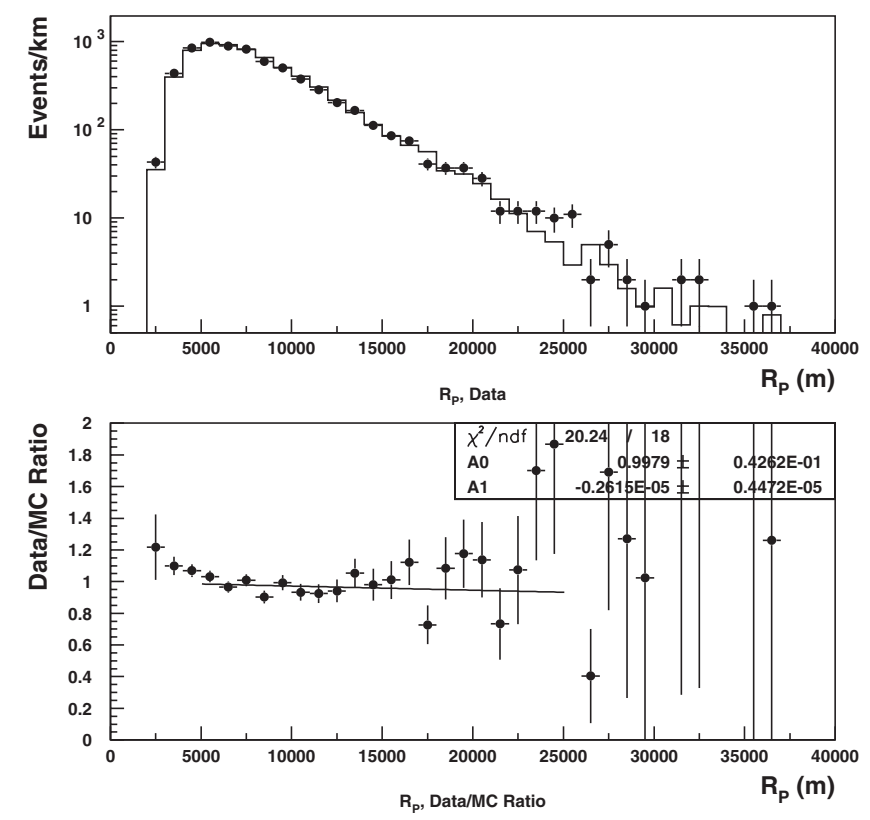

Figure 1. Data/MC comparison of impact parameter at HiRes-II. Shown below the main comparison is the ratio of data to MC.

speed of light, one can show that the time of signals reaching the detector should have the form

$$
t_{i}-t_{0}=\frac{R_{p}}{c} \tan \frac{\pi-\psi-\chi_{i}}{2}
$$

where $t_{i}$ is the measured time of a signal coming from an angle $\chi_{i}$ along the SDP (measured from the horizon). The fit parameters are $R_{p}$, the impact parameter of the shower on the detector, $\psi$, the angle the shower makes with respect to the ground in the SDP and $t_{0}$, the earliest possible time for a signal to reach the detector (it is also the time when the shower reaches the point of closest approach to the detector).

Unless the observed track has a very great extent in $\chi$ the three parameters are highly correlate leading to poorer resolution of the shower geometry within the SDP plane than of the SDP itself. Nonetheless, monocular analysis is still useful as it will have a considerably larger aperture for detecting UHECR air showers at high energies and will have a much lower low-energy threshold.

With the geometry determined, the shower profile is reconstructed. To determine the best estimate of shower development parameters, we used the so-called Inverse MC (IMC) method. In this technique, shower development is assumed to follow the Gaisser-Hillas profile, and the $N_{\max }$ and $X_{\max }$ parameters are varied to find the best fit. The name of the technique comes from the fact that for each set of GH parameters, the same detector simulation program is used to find the expected PMT signals as is used in calculating the overall detector aperture. The fit is performed by comparing individual PMT responses between data and IMC predictions.

The aperture of fluorescence detectors changes with energy. This is an advantage as it allows for very large apertures at the highest energies. It is also a challenge as there is no way to determine the aperture geometrically, it must be estimated by MC simulation. The MC simulation, however, can be made robust by requiring the simulation to produce distributions of observables which can then be compared to data distributions. The accuracy of the MC simulations is as good as data/MC comparisons can be made to be. 


\section{UHECR 2012}

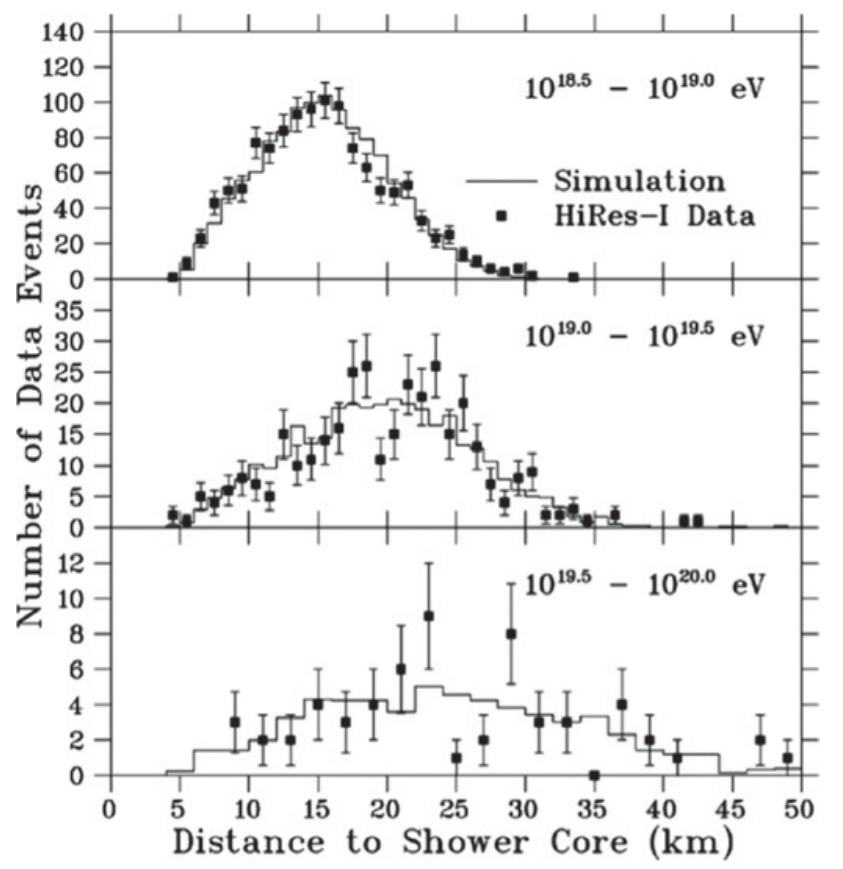

Figure 2. Data/MC comparison of the core distance on the ground at HiRes-I. The comparison is shown in three energy bands to emphasize how the shape changes and that the MC reproduces these changes.
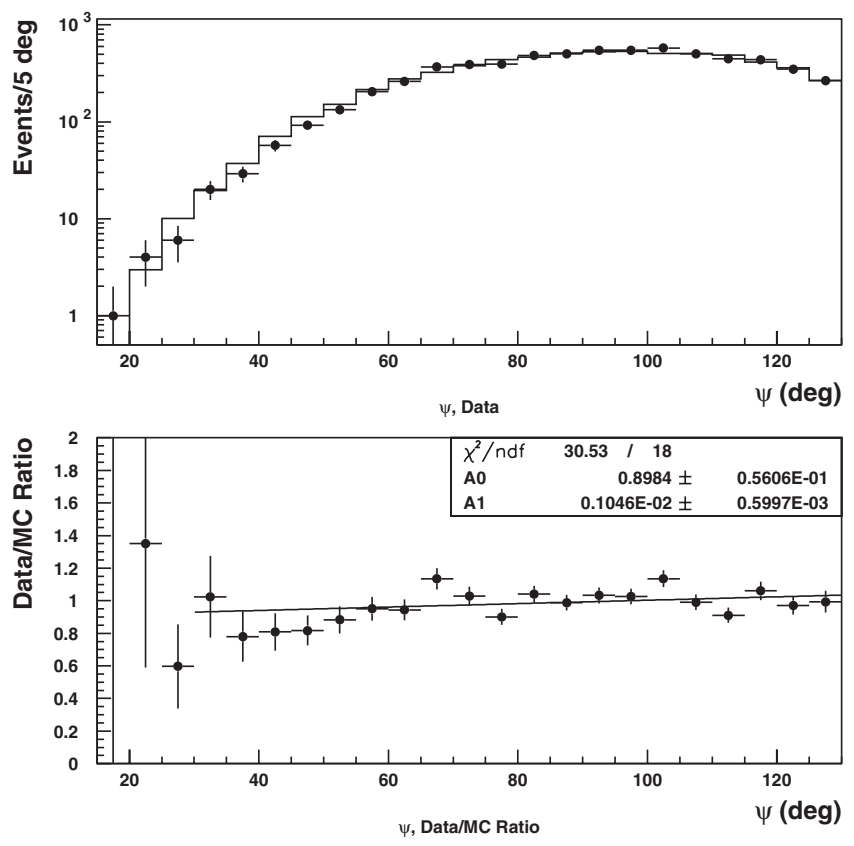

Figure 3. Data/MC comparison of $\psi$ at HiRes-II. Shown below the main comparison is the ratio of data to MC. 
EPJ Web of Conferences

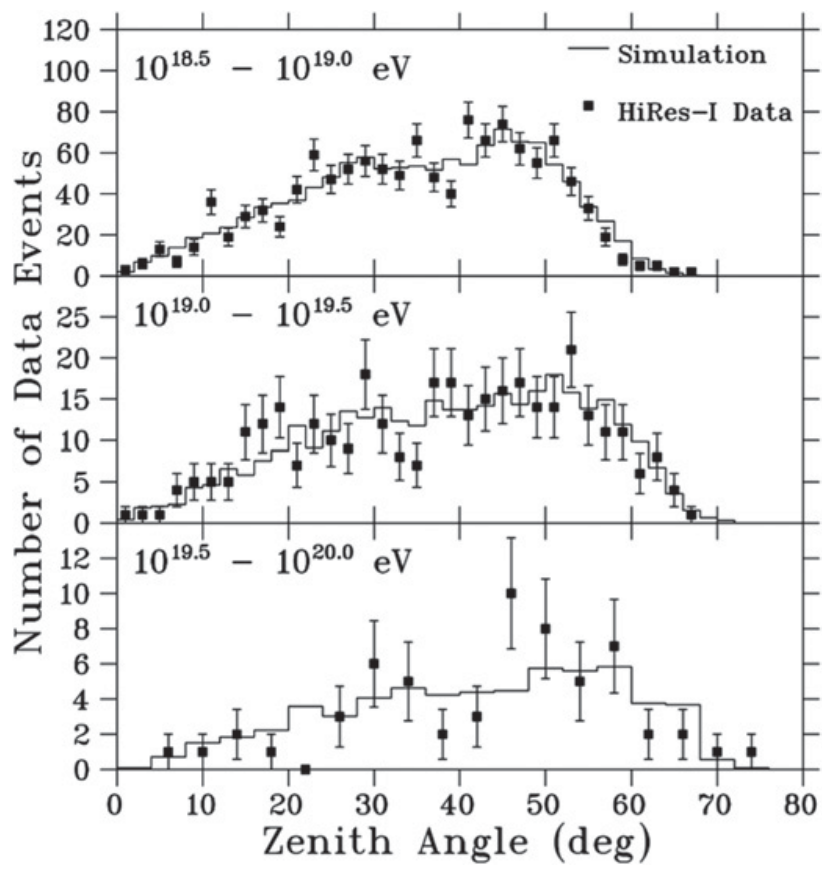

Figure 4. Data/MC comparison of the zenith angle at HiRes-I. The comparison is shown in three energy bands.
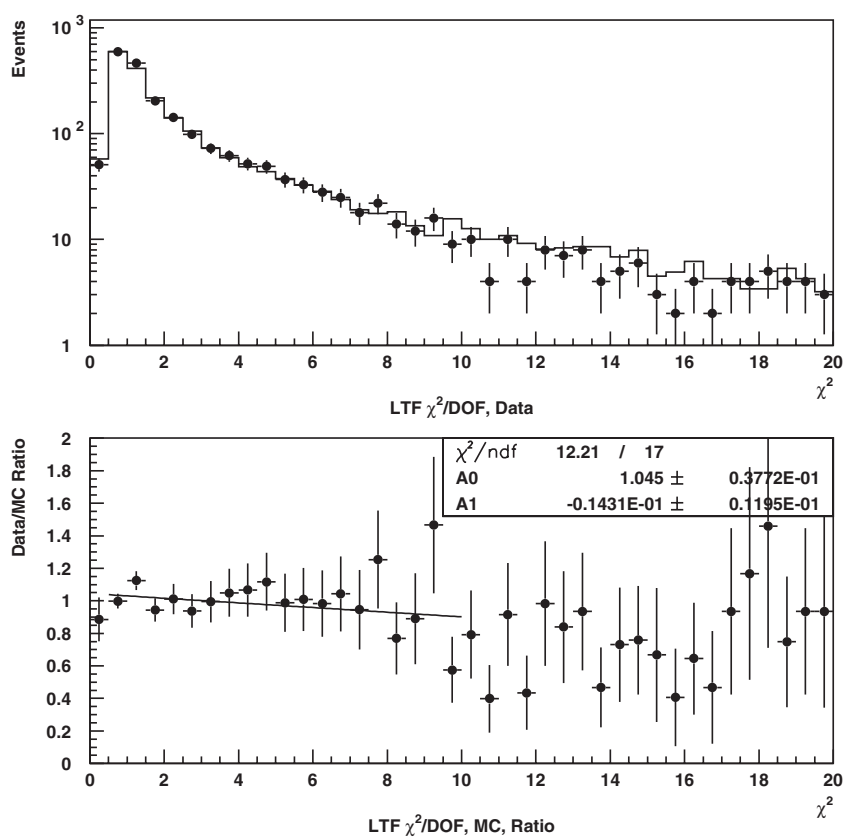

Figure 5. Data/MC comparison of the linear time fit $\chi^{2}$ at HiRes-II. 


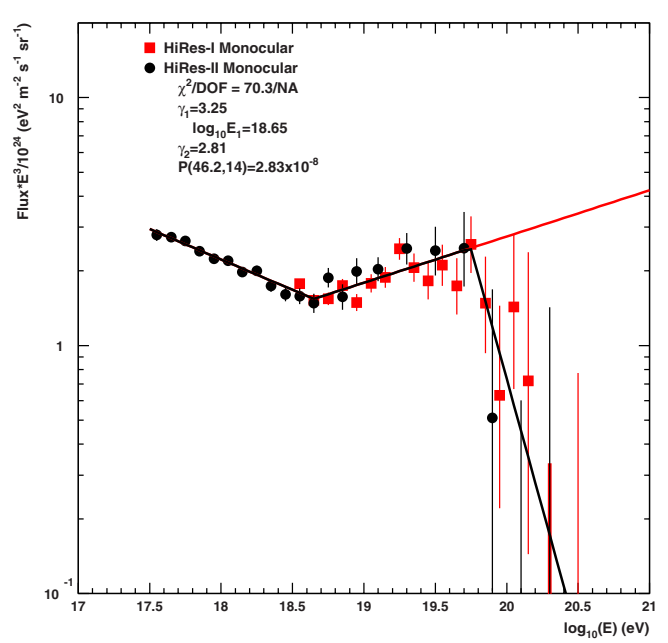

Figure 6. The UHECR spectrum as measured by HiRes-I and HiRes-II including a broken power-law fit.

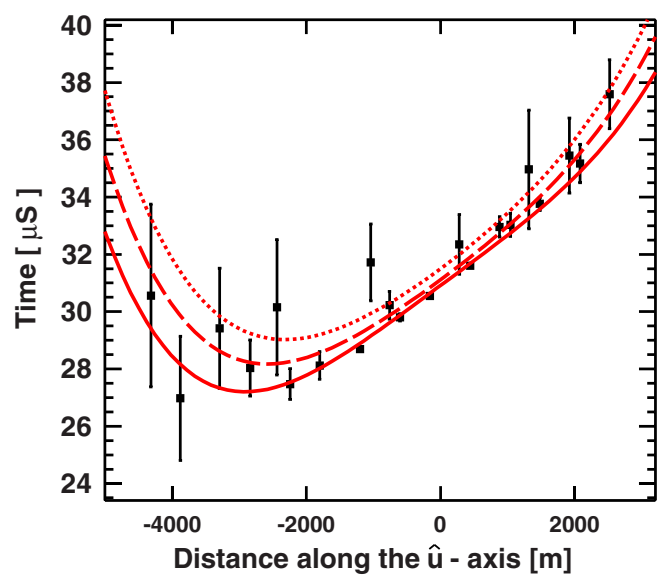

Figure 7. An example fit of an event showing time vs counter position. The counter position is projected onto the shower direction projected onto the ground.

We compare variables which impinge directly on the aperture: the distance to showers and their angular distribution. Figure 1 shows the dataa4/MC comparison of the impact parameter at HiRes-II. Figure 2 shows a comparison of the distance on the ground between the detector and the shower impact point in three energy bands for HiRes-I. Figure 3 shows the distribution of $\psi$ at HiRes-II. Figure 4 shows the distribution of zenith angles at HiRes-I. Finally, Figure 5 shows the agreement in the fit $\chi^{2}$ distribution at HiRes-II. Agreement in the fit quality implies that the resolution measurements made in the MC simulation are applicable to the data.

After calculating the aperture of the experiment as a function of energy, it is straightforward to calculate the spectrum. The spectrum of UHECRs as measured by both HiRes-I and HiRes-II is shown in Figure 6. HiRes was the first experiment to observe the GZK Cutoff. The significance of the cutoff at $10^{19.75} \mathrm{eV}$ is determined by counter the number of events observed above this energy and comparing to the number of events expected from an extension of the measured power law from energies below the break to energies above the break. In the case of HiRes, after correcting for the overlap of the two 


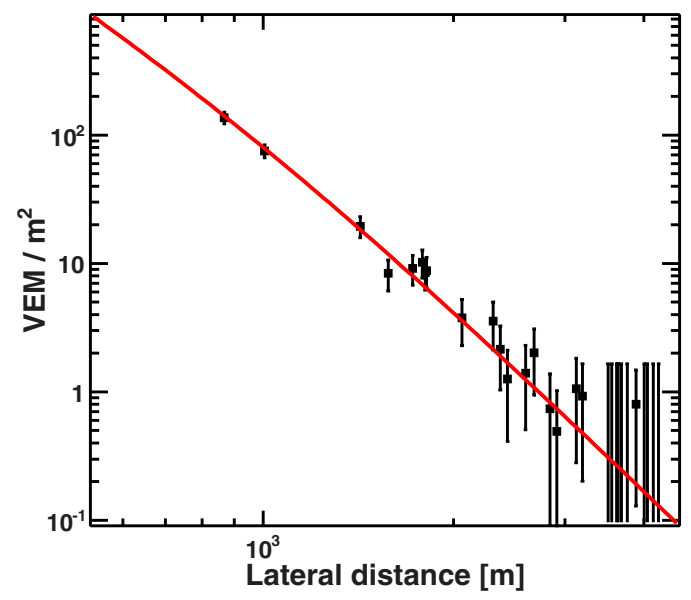

Figure 8. A fit to the LDF. The value of the fit at $800 \mathrm{~m}$ is used in determining the shower energy.

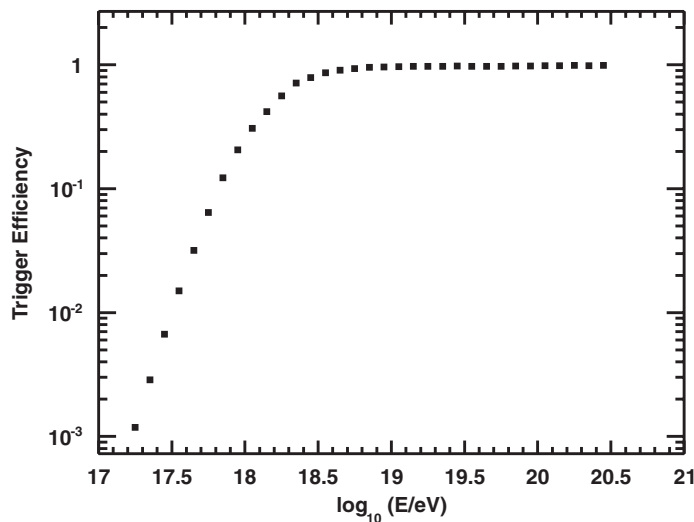

Figure 9. The efficiency of the TA SD array as a functino of energy. Energies above $10^{18.2} \mathrm{eV}$ are used in the analysis, corresponding to efficiencies above $8 \%$.

monocular apertures, 13 events were observed while 43 events were expected in the case of no break. This corresponds to a $5.3 \sigma$ result.

The position of the cutoff as measured by HiRes can be qualified by finding the energy at which the integral spectrum falls to half of what would have been expected without the cutoff. For HiRes the log of this energy is $19.73 \pm 0.07$ agreeing nicely with the prediction of Berezinsky et al [3] of 19.72. This results assumes that the bulk of UHECR primaries are protons, in agreement with all HiRes composition measurements.

\section{TELESCOPE ARRAY SURFACE DETECTOR MEASUREMENTS}

The Telescope Array (TA) is hybrid detector currently operating west of Delta, UT. It consists of 507 surface detectors (SDs) on a square grid with spacing $1.2 \mathrm{~km}$ overlooked by three sets of fluorescence telescopes. It has been operated continuously since 2007. The TA Collaboration is made up of scientists from the United States, Japan, South Korea, Russia, and Belgium and includes many former members 


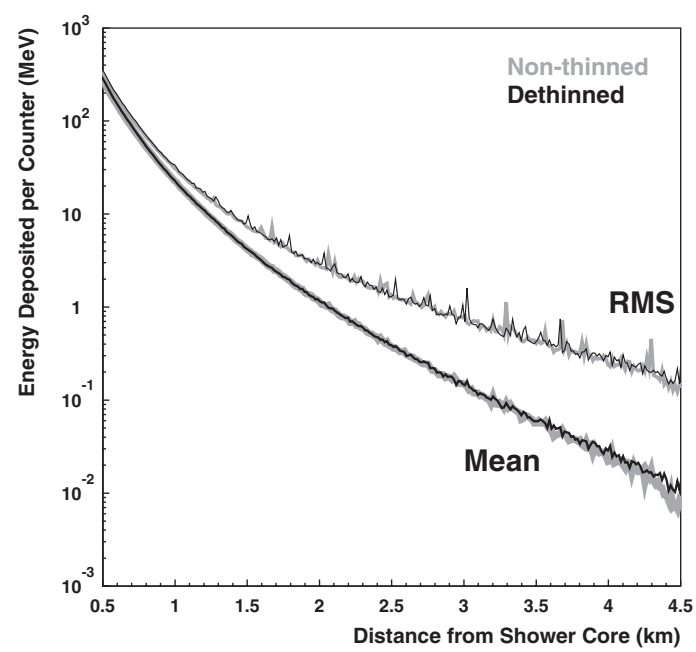

Figure 10. The mean energy deposit as a function of distance to the shower core and the RMS of the energy deposit distribution. The results of a Corsika run with no thinning (grey) are compared to the results of a Corsika run with thinning which has been subject to a dethinning preceedure.

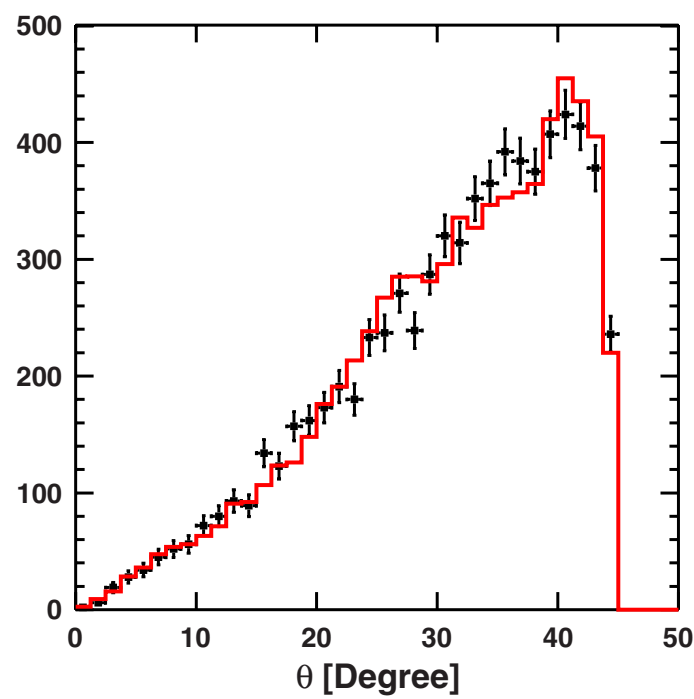

Figure 11. Data/MC comparison of the zenith angle distribution.

of the HiRes and AGASA collaborations. The detector is described in greater detail elsewhere $[4,5]$. The SDs each have an active area of $3 \mathrm{~m}^{2}$, consisting of two layers of $1-\mathrm{cm}$ thick scintillator. The light from the scintillators are collected by wave-shifting fibers to photomultiplier tubes, one each for the top and bottom sections. The three FD sites each contain two rings of mirrors, viewing $3^{\circ}-31^{\circ}$ in elevation, and views $\sim 110^{\circ}$ in azimuth. The azimuthal fan from the three sties are arranged to fully cover the SD array at energies above $10^{19} \mathrm{eV}$.

The results presented here are mainly from the SD array operating on its own. However, the energy scale normalization comes from events seen in tandem with all three of the FD sites. It should be 


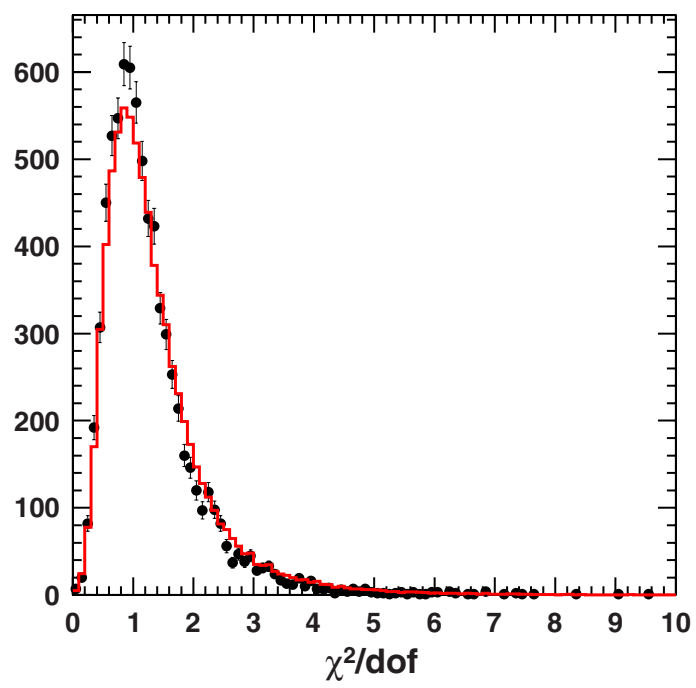

Figure 12. The distribution of $\chi^{2}$ of the LDF fit. The good comparison implies that the resolution calculations are aso good.

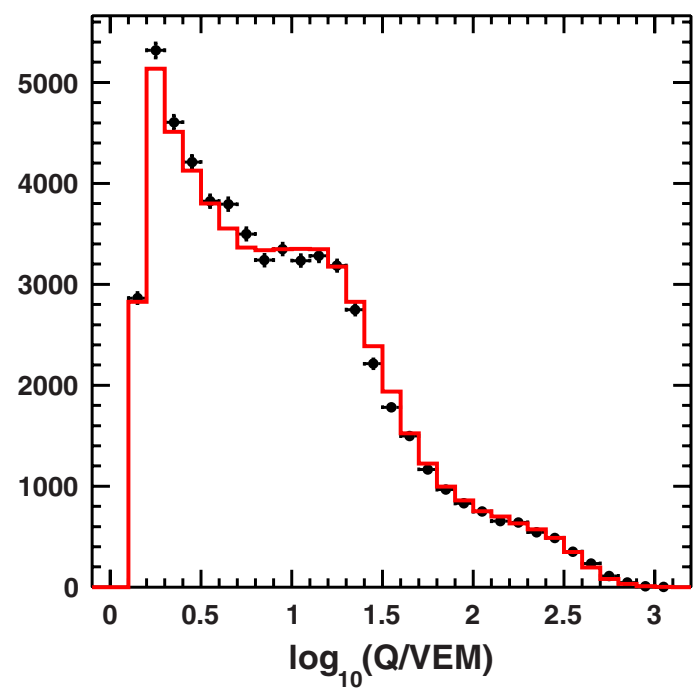

Figure 13. Data/MC comparison of the distribution of energy deposits per counter in all counters.

emphasized that the energy normalization does not come solely from the Middle Drum site which uses refurbished HiRes mirrors and PMTs.

Analysis of the SD events begins by finding the barycentric average of the hit counters. The space and time distribution is then combined in a fit to find the most likely core position and primary cosmic ray direction. An example of the time of each counter vs counter position is shown in Figure 7. Once the core and direction are found, the charge particle lateral distribution is fit to determine the density of charged particles $800 \mathrm{~m}$ from the shower core, $S(800)$. $S(800)$ is the primary energy-dependent variable used in TA SD analyses. An example LDF fit is shown in Figure 8.

The SD aperture, unlike the case in HiRes is nearly constant above some threshold energy, defined by the area of the array and the angular range of acceptance. However, the range of useful energies 


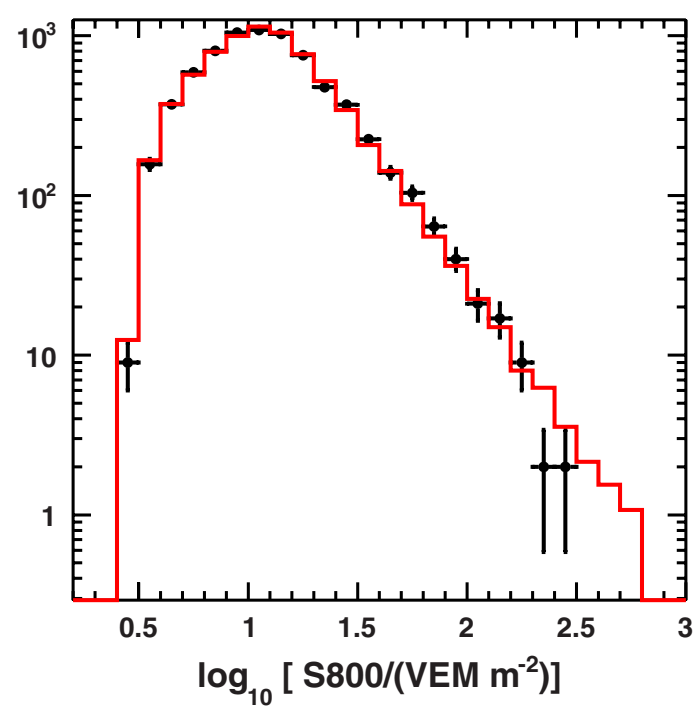

Figure 14. Data/MC comparison of $S(800)$ values.

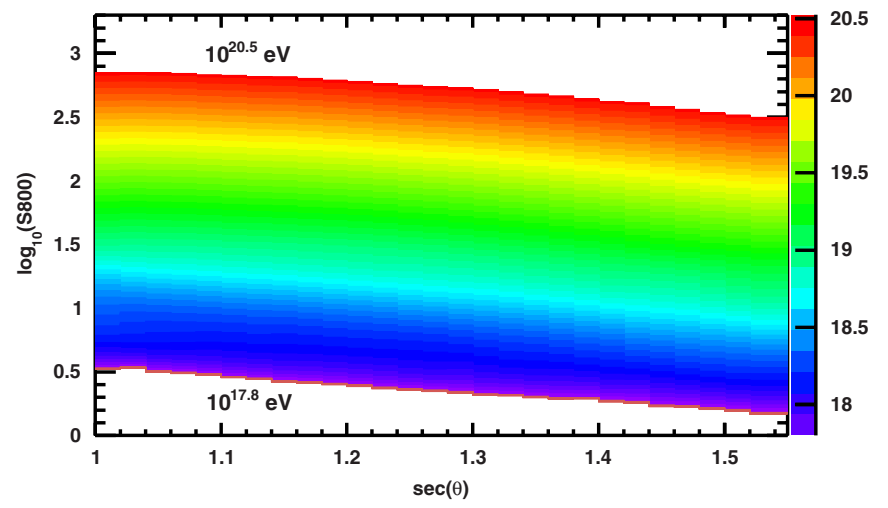

Figure 15. The look-up table used to estimate the energy of an air-shower based on its zentih angle an $S(800)$ measurement.

can be considerably extended by careful estimation of the acceptance efficiency at lower energies. Figure 9 shows the calculated efficiency of the SD array. It is the result of careful MC simulation like the fluorescence case, with verification of the simulation through data/MC comparisons. This is the first time such a thoroughgoing use of the data/MC comparison method has been performed in an SD spectrum measurement. To generate the required size MC sample requires the use of thinning during simulation of showers. The statistical effects of thinning are removed by a de-thinning proceedure [6]. The result of the dethinning preceedure are compared with the same shower generated with no thinning as shown in Figure 10.

We show data/MC comparisons which impact the efficiency directly: zenith angle, LDF $\chi^{2}$, energy deposit per counter and $S(800)$. These are shown in Figures 11-14.

The calculation of shower energy from $\mathrm{S}(800)$ does not follow the traditional constant-intensity-cut method but rather uses a look-up table derived from the MC simulation. The data/MC comparisons validate the look-up table in much the same manner as they validate the acceptance calculation. The 


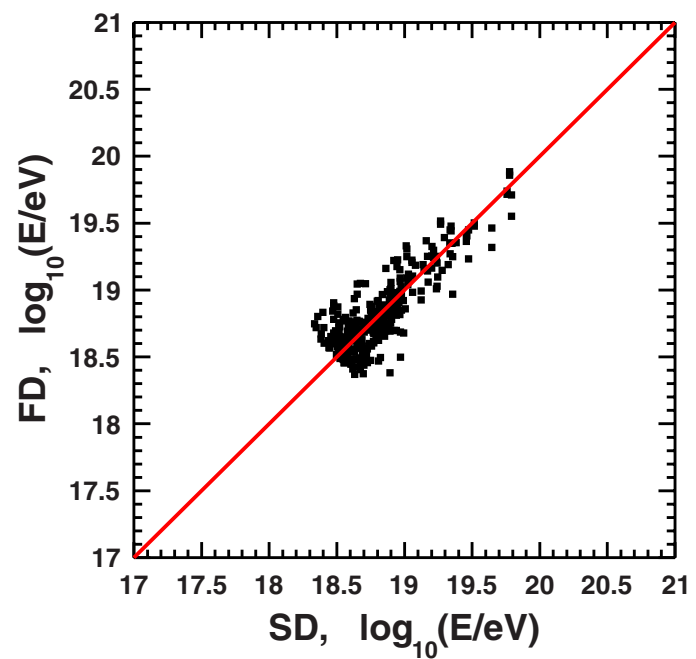

Figure 16. Comparison of rescaled SD energies with energies measured by the FD detectors.

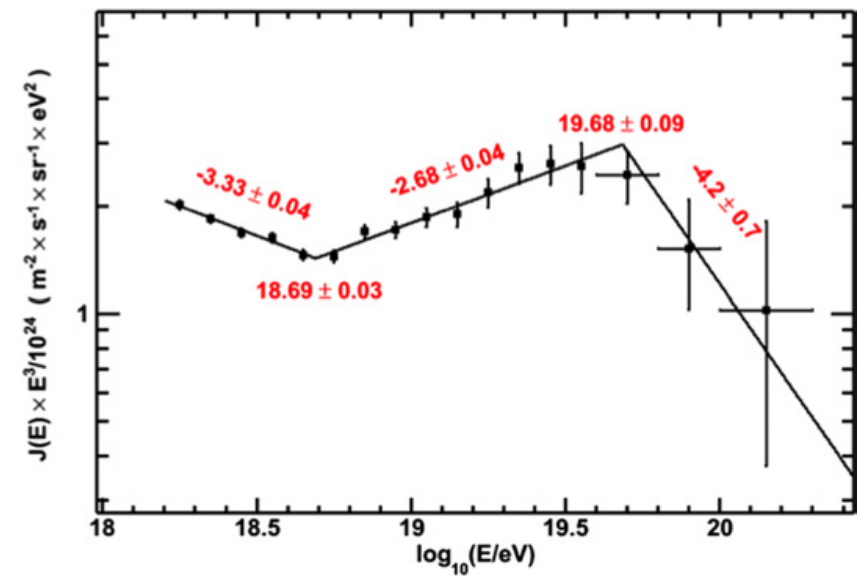

Figure 17. The spectrum of UHECRs as measured by the TA surface detector. The spectrum is fit to a broken power law, with the spectral slopes and break points as noted.

look-up table is displayed in Figure 15. Finally, the energy is scaled to match the FD energy scale by comparing the energies of events seen by both the SD and any (and all) of the three FD sites. A scatter plot of the SD and FD energies (after the SD energy re-scaling) is shown in Figure 16.

Finally, we show the spectrum as calcaulted by the TA surface detectors in Figure 17. This is the largest exposure measurement available from TA at this time. The spectrum agrees very well with the HiRes measurements present above as shown in Figure 18. This agreement is especially notable given the different detection techniques involved, fluorescence in the case of HiRes, surface detectors in the case of TA. What is common to the two measurements is the thoroughgoing use of the Data/MC comparison method of validating the MC simulation necessary for robust estimates of aperture, acceptance and energy. 


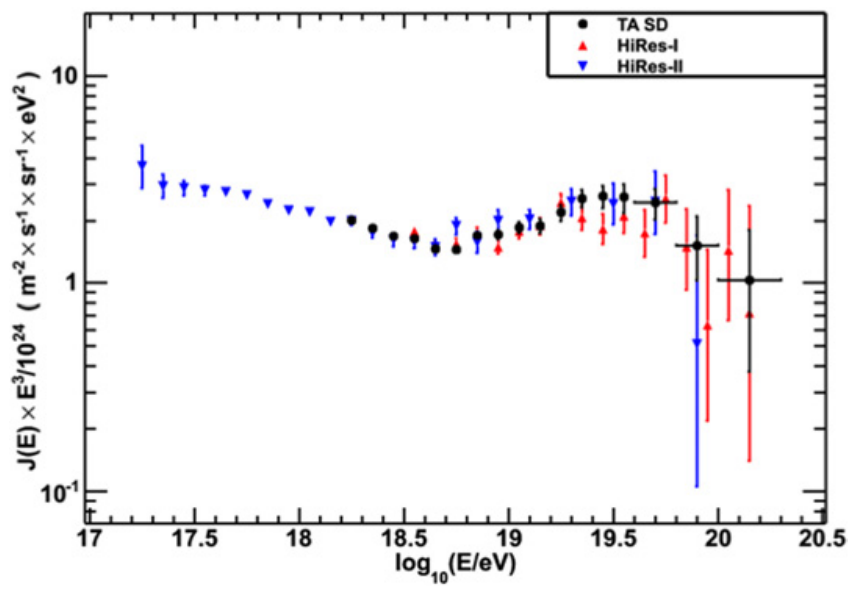

Figure 18. A compilation of the HiRes monocular and TA SD spectrum measurements highlighting the close agreement between the measurements.

\section{References}

[1] Abu-Zayyad et al. Proceedings of the 26th ICRC (Salt Lake City) (1999) vol. 5, p. 349

[2] J. H. Boyer et al., Nucl. Inst. Meth. A 482, 457 (2002)

[3] V. Berezinsky et al., Phys. Rev. D 74, 0403005 (2006), [hep-ph/0204357]

[4] H. Tonuno et al., Nucl. Inst. Meth. A 676 (2012)

[5] T. Abu-Zayyad et al., arXiv:1201.4964

[6] B. T. Sotkes et al., Astropart. Phys. 35 (2012) 